\title{
Weak dipole moments of the top quark and flavor violation
}

\section{B. Quezadas-Vivian*}

Facultad de Ciencias Físico Matemáticas, Universidad Michoacana de San Nicolás de Hidalgo

E-mail: brendysqv19@hotmail.com

\author{
J. I. Aranda ${ }^{1}$, J. Montaño ${ }^{1,2}$, F. Ramírez-Zavaleta ${ }^{1}$, E. S. Tututi ${ }^{1}$ \\ ${ }^{1}$ Facultad de Ciencias Físico Matemáticas, Universidad Michoacana de San Nicolás de \\ Hidalgo, Avenida Francisco J. Mújica S/N, 58060 Morelia, Michoacán, México \\ ${ }^{2}$ CONACYT, Av. Insurgentes Sur 1582, Col. Crédito Constructor, Benito Juárez, C. P. 03940, \\ Ciudad de México, México. \\ E-mail: jarandas@umich.mx, j.montano.d@hotmail.com, \\ feryuphy@yahoo.com.mx, tututi@umich.mx
}

\begin{abstract}
We calculate the weak electromagnetic static properties for any charged fermion of the Standard Model (SM) in the context of a general effective extended vector neutral current model with flavor changing $\bar{f}_{i} f_{j} Z^{\prime}$ vertices. In this work we give phenomenological results for the weak electromagnetic properties of the quark top. We use known theoretical constraints coming from various $Z^{\prime}$ models to bind the effective coupling parameters, and we compare our results with the theoretical prediction of the $Z$ from the SM. The experimental detection of non zero anomalous weak electromagnetic dipole moments of heavy fermions, at the current sensitivity, would be a clear evidence of new physics beyond the SM.
\end{abstract}

7th Annual Conference on Large Hadron Collider Physics - LHCP2019

20-25 May, 2019

Puebla, Mexico

* Speaker. 


\section{Introduction}

The study of the flavor violation has regained interest due to the discovery of the neutrino oscillations [1,2], which shows that the flavor conservation is violated in the Nature, this justifies to study processes that violates flavor as possible precursors of new theories that might explain more deeply the character of the elementary particles.

The known experimental SM precision measurements of anomalous magnetic moments for elementary charged particles are known only for the electron and the muon [3, 4], while there still not exist experimental measures for the tau and the quarks, these dipole moments have long received considerable attention, which has been boosted in recent years due to the significant progress in the experimental area as the study of the static electromagnetic properties of fermions provides a unique opportunity to search for effects of new physics, and beyond of the electromagnetic properties of a fermion, there is also great interest in its static weak properties, which are associated with the $Z$ boson interaction, which we carry out in this work. This keeps open the investigation field on the fermions static weak electromagnetic dipole moments and its relation with possible new physics effects regarding to the flavor changing violation.

The anomalous weak-magnetic moment of fermions carries important information about their interactions with other particles. It may be seen as the coefficient of a chirality-flipping term in the effective Lagrangian of the $\mathrm{Z}$ coupled to fermions. Therefore, at $q^{2} \neq 0$, it is expected to be proportional to the mass of the fermion, and only heavy fermions (leptons or quarks) are good candidates to have a measurable anomalous weak magnetic moment [5].

\section{The Extended Model}

The most simple extended model that predicts the existence of an extra weak neutral gauge boson, identified as $Z^{\prime}$, is that based on the extended electroweak gauge group $S U_{L}(2) \times U_{Y}(1) \times$ $U^{\prime}(1)$, which once implemented in the spontaneous symmetry breaking (SSB) give rises to mixings between the SM gauge boson $Z$ and the $Z^{\prime}$. [6].

We use the most general renormalizable effective Lagrangian, which includes the fermion flavor violation mediated by a new neutral massive gauge boson $Z^{\prime}$, coming from any extended or grand unification model [6]:

$$
\mathscr{L}_{N C}=\sum_{i, j}^{3}\left[\bar{f}_{i} \gamma^{\alpha}\left(\Omega_{L f i f j} P_{L}+\Omega_{R f i f j} P_{R}\right) f_{j}+\bar{f}_{j} \gamma^{\alpha}\left(\Omega_{L f j f i}^{*} P_{L}+\Omega_{R f j f i}^{*} P_{R}\right) f_{i}\right] Z_{\alpha}^{\prime},
$$

where $f_{i}$ is any fermion of the $\mathrm{SM}, P_{L, R} \equiv \frac{1 \mp \gamma_{5}}{2}$ are the chiral projectors, and $Z_{\alpha}^{\prime}$ is the new neutral massive gauge boson predicted by several extensions of the SM. The $\Omega_{L, R f_{i} f_{j}}$ parameters represent the strength of the $f_{i} f_{j} Z^{\prime}$ coupling. We will assume that $\Omega_{L f_{i} f_{j}}=\Omega_{L f_{j} f_{i}}$ and $\Omega_{R f_{i} f_{j}}=\Omega_{R f_{j} f_{i}}$. The Lagrangian includes both flavor-conserving and flavor-violating couplings. The flavor-conserving couplings, $Q_{L, R}^{f_{i}}$ [6], are related to the $\Omega$ couplings as $\Omega_{L f_{i} f_{i}}=-g_{2} Q_{L}^{f_{i}}$ and $\Omega_{R f_{i} f_{i}}=-g_{2} Q_{R}^{f_{i}}$, where $g_{2}=\frac{g}{\cos \theta_{W}}$ is the gauge coupling of the $Z^{\prime}$ boson [6] . 


\section{Static Weak Electromagnetic Dipole Moments}

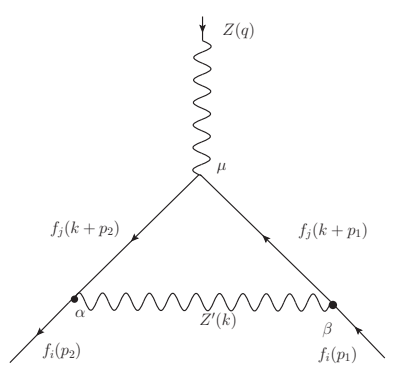

Figure 1: 1-loop contribution to the static weak electromagnetic dipole moments induced by a $Z^{\prime}$ with flavor changing.

The general vertex Lorentz structure is [7]

$$
i e \bar{u}\left(p^{\prime}\right) \Gamma^{\mu} u(p)=i e \bar{u}\left(p^{\prime}\right)\left\{i \sigma^{\mu v} q_{v}\left[F_{M}\left(q^{2}\right)-i F_{E}\left(q^{2}\right) \gamma^{5}\right]\right\} u(p)
$$

where $F_{M}$ is the weak magnetic and $F_{E}$ is the weak electric form factor, and the tensor amplitude $\mathscr{M}_{f i}^{\mu}=\bar{u}\left(p^{\prime}\right) \Gamma^{\mu} u(p)$. The static properties arise when the $\mathrm{Z}$ boson is on-shell: $q^{\mu} \varepsilon_{\mu}=0$ and $q^{2}=m_{Z}^{2}$. Hence the static anomalous magnetic, $\mu_{f i}^{w}$, and electric, $d_{f i}^{w}$, weak dipole moments are

$$
F_{M}\left(m_{Z}^{2}\right) \equiv-\frac{\mu_{f_{i}}^{w}}{2 m_{f_{i}}}, \quad \quad F_{E}\left(m_{Z}^{2}\right) \equiv-\frac{d_{f_{i}}^{w}}{e}
$$

The contribution of the $f_{i} f_{j} Z^{\prime}$ couplings to the static weak electromagnetic dipole moments at 1-loop level is depicted in Fig. 1., whose amplitude is

$$
\begin{aligned}
\mathscr{M}_{f_{i}}^{\mu}=- & i^{6} \frac{g}{2 c_{W}} \int \frac{d^{4} k}{(2 \pi)^{4}} \bar{u}\left(p^{\prime}\right)\left[\gamma^{\alpha_{1}}\left(g_{V Z^{\prime}}^{f_{i} f_{j}}-g_{A Z^{\prime}}^{f_{i} f_{j}} \gamma^{5}\right)\right]\left(k+\not p^{\prime}+m_{f_{j}}\right) \gamma^{\mu}\left(g_{V Z}^{f_{j}}-g_{A Z}^{f_{j}} \gamma^{5}\right) \\
& \times \frac{\left(k+\not p+m_{f_{j}}\right)\left[\gamma^{\alpha_{2}}\left(g_{V Z^{\prime}}^{f_{i} f_{j^{*}}}-g_{A Z^{\prime}}^{f_{i} f_{j} *} \gamma^{5}\right)\right]}{\left(k^{2}-m_{Z^{\prime}}^{2}\right)\left[\left(k+p^{\prime}\right)^{2}-m_{f_{j}}^{2}\right]\left[(k+p)^{2}-m_{f_{j}}^{2}\right]} u(p)\left(-g_{\alpha_{1} \alpha_{2}}+\frac{k_{\alpha_{1}} k_{\alpha_{2}}}{m_{Z^{\prime}}^{2}}\right) .
\end{aligned}
$$

The integral must be solved applying tensor decomposition method.

The static weak dipole moments have two scenarios for the CP property: i) CP conserving which only gives rise to $\mu_{f_{i}}^{w}$, while forbids $d_{f_{i}}^{w}$;ii) CP violation that gives rise to both $\mu_{f_{i}}^{w}$ and $d_{f_{i}}^{w}$.

The weak anomalous magnetic moment is:

$$
\begin{aligned}
& \mu_{f_{i}}^{w}=g_{V f_{j}}^{Z}\left\{\left|g_{V f_{i}}^{Z^{\prime}}\right|^{2} F_{V}^{a}\left(m_{f_{i}}, m_{f_{j}}, m_{Z^{\prime}}\right)+\left|g_{A f_{i}}^{Z^{\prime}}\right|^{2} F_{A}^{a}\left(m_{f_{i}}, m_{f_{j}}, m_{Z^{\prime}}\right)\right\} \\
& +g_{A f_{j}}^{Z}\left\{g_{A f_{i}}^{Z^{\prime}} g_{V f_{i}}^{Z^{\prime} *}+g_{A f_{i}}^{Z^{\prime} *} g_{V f_{i}}^{Z^{\prime}}\right\} F_{V A}^{a}\left(m_{f_{i}}, m_{f_{j}}, m_{Z^{\prime}}\right)
\end{aligned}
$$

where

$$
\begin{aligned}
& \left|g_{V f i}^{Z^{\prime}}\right|^{2}=\frac{1}{4}\left[\left(\operatorname{Re} \Omega_{L f_{i} f_{j}}+\operatorname{Re} \Omega_{R f_{i} f_{j}}\right)^{2}+\left(\operatorname{Im} \Omega_{L f_{i} f_{j}}+\operatorname{Im} \Omega_{R f_{i} f_{j}}\right)^{2}\right], \\
& \left|g_{A f i}^{Z^{\prime}}\right|^{2}=\frac{1}{4}\left[\left(\operatorname{Re} \Omega_{L f_{i} f_{j}}-\operatorname{Re} \Omega_{R f_{i} f_{j}}\right)^{2}+\left(\operatorname{Im} \Omega_{L f_{i} f_{j}}-\operatorname{Im} \Omega_{R f_{i} f_{j}}\right)^{2}\right] .
\end{aligned}
$$


The weak electric dipole moment is:

$$
d_{f_{i}}^{w}=g_{V f_{j}}^{Z}\left\{g_{A f_{i}}^{Z^{\prime}} g_{V f_{i}}^{Z^{\prime} *}-g_{V f_{i}}^{Z^{\prime}} g_{A f_{i}}^{Z^{\prime} *}\right\} F_{V A}^{d}\left(m_{f_{i}}, m_{f_{j}}, m_{Z^{\prime}}\right),
$$

where

$$
g_{A f_{i}}^{Z^{\prime}} g_{V f_{i}}^{Z^{\prime} *}-g_{V f_{i}}^{Z^{\prime}} g_{A f_{i}}^{Z^{\prime} *}=i\left(\operatorname{Re} \Omega_{L f_{i} f_{j}} \operatorname{Im} \Omega_{R f_{i} f_{j}}-\operatorname{Re} \Omega_{R f_{i} f_{j}} \operatorname{Im} \Omega_{L f_{i} f_{j}}\right) .
$$

$F_{V}^{a}, F_{A}^{a}, F_{V A}^{a}$ and $F_{V A}^{d}$ are extended form factors that depend of $m_{f_{i}}, m_{f_{j}}$ and $m_{Z^{\prime}}$.

\section{Results: the top weak electromagnetic moments}

We carry out the phenomenological analysis on the top weak electromagnetic moments by considering the different $Z^{\prime}$ gauge bosons, $Z_{s}^{\prime}, Z_{L R}^{\prime}, Z_{\chi}^{\prime}, Z_{\psi}^{\prime}$ and $Z_{\eta}^{\prime}$, whose coupling parameters, $\Omega_{L, R}$, were computed in Ref.[6]
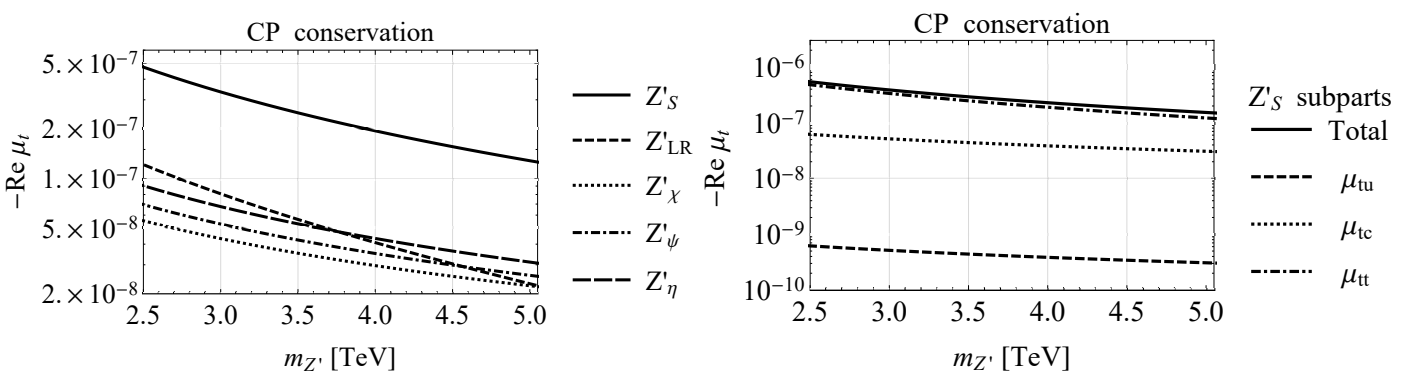

Figure 2: Anomalous weak magnetic dipole moment of the top with $\mathrm{CP}$ conserving, and main contribution due the $Z_{S}^{\prime}$ and its subparts.
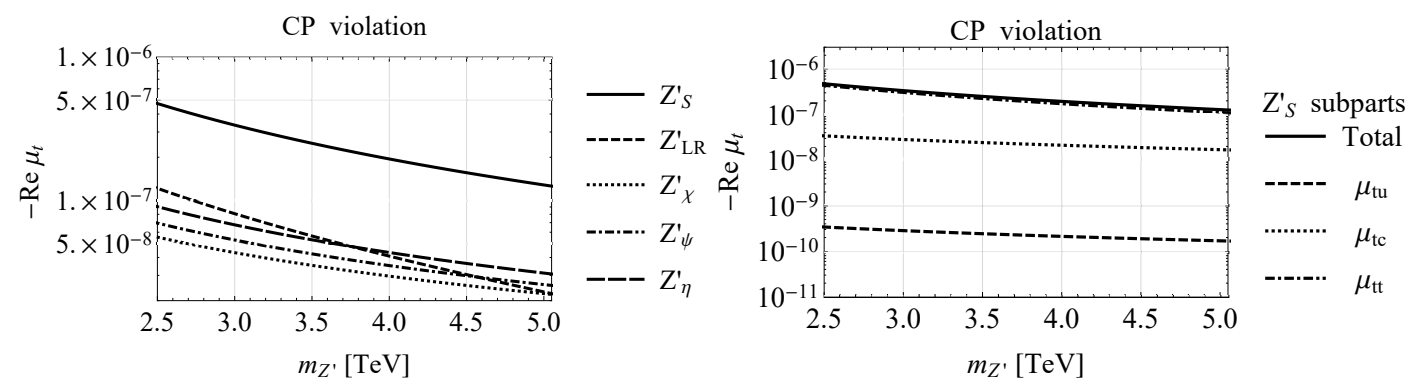

Figure 3: Anomalous weak magnetic dipole moment of the top with $\mathrm{CP}$ violating and main contribution due the $Z_{S}^{\prime}$ and its subparts.

The Fig. 2 shows the real part of the anomalous weak magnetic dipole moment of the top in the CP conserving scenario, as a funtion of the $Z^{\prime}$ gauge boson mass, for the interval $m_{Z^{\prime}}=[2.5,5] \mathrm{TeV}$, and shows too the main contribution due the $Z_{s}^{\prime}$ and its subparts which provides the higher signal.

The Fig. 3 shows the real part of the anomalous weak magnetic dipole moment of the top in the CP violation scenario. From this plot we appreciate that $Z_{s}^{\prime}$ provides the higher signal and shows too its subparts, while the smallest one corresponds to $Z_{\chi}^{\prime}$. 


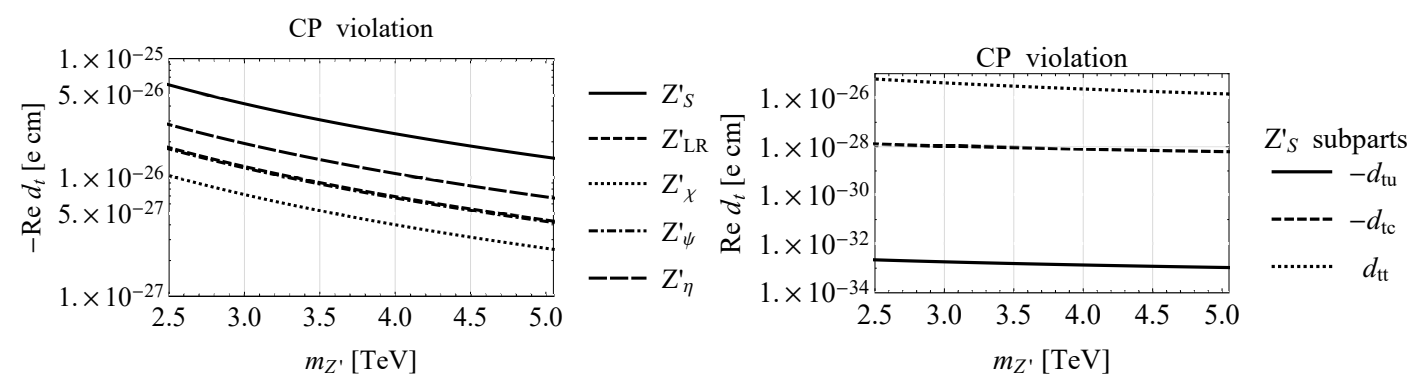

Figure 4: Anomalous weak electric dipole moment of the top and main contribution due the $Z_{S}^{\prime}$ and its subparts.

The Fig. 4 shows the real part of the weak electric dipole moment of the top in the CP violation scenario, where the models are between the order of magnitude of $\sim 10^{-26} \mathrm{ecm}$ and $\sim 10^{-27} \mathrm{ecm}$, and shows the main contribution which provides the higher signal.

The theoretical SM contribution to the weak dipole magnetic moment of the on-shell top is $\mu_{t_{S M}}=$ $-1.15 \times 10^{-3}-i 7.21 \times 10^{-20}$, and with respect to this value we can compare our results, shown in Figs. 2 and 3. On the other hand, the value at large momentum transfer $\sqrt{q^{2}}=500 \mathrm{GeV}$ is $\mu_{t_{S M}}=-2.46 \times 10^{-4}-$ $i 1.45 \times 10^{-3}[9]$.

\section{Conclusions}

The new physics effects due to possible presence of flavor changing neutral currents mediated by a new neutral massive gauge boson, identified as $Z^{\prime}$, have been studied on the weak electromagnetic moments of the top quark, obteined anallyticaly the static weak electromagnetic dipole moments for any charged fermion of the SM.

For the $\mu_{t}\left(Z^{\prime}\right)$ we have found signatures in the range of $\sim 10^{-7}$ and $\sim 10^{-8}$, for both CP conserving and violation scenarios, which is barely 4 orders of magnitude lower than the $\operatorname{SM} \mu_{t}(S M) \sim 10^{-3}$.

For the $d_{\tau}\left(Z^{\prime}\right)$ we predict signatures of $\sim 10^{-26} \mathrm{ecm}$ and $\sim 10^{-27} \mathrm{ecm}$.

The $Z^{\prime}$ provides promising predictions which might shed light on new physics effects.

\section{Acknowledgments}

This work has been partially supported by SNI-CONACYT and CIC-UMSNH. J.M. thanks the Catedras CONACYT Program support.

\section{References}

[1] R. Becker-Szendy et al., Nucl. Phys. Proc. Suppl. 38, 331 (1995); Y. Fukuda et al., Phys. Lett. B 335, 237 (1994); Phys. Rev. Lett. 81, 1562 (1998); H. Sobel, Nucl. Phys. Proc. Suppl.91, 127 (2001); M. Ambrossio et al., Phys. Lett. B 566, 35 (2003); M. Apollonio et al., Eur. Phys. J. C 27, 331 (2003); M. B. Smy et al., Phys. Rev. D 69, 011104(R) (2004); S.N. Ahmed et al., Phys. Rev. Lett. 92, 181301 (2004); Y. Ashie et al., Phys. Rev. Lett. 93, 101801 (2004); E. Aliu et al., Phys. Rev. Lett. 94, 081802 (2005); Y. Ashie et al., Phys. Rev. D 71, 112005 (2005); W. W. M. Allison et al., Phys. Rev.D 72, 052005 (2005); P. Adamson et al., Phys. Rev. D 73, 072002 (2006).

[2] B. Odom, D. Hanneke, B D’urso and Gabrielse, Phys. Rev. Lett. 97, 030801 (2006).

[3] G.W. Bennett et al., Phys. Rev. D73, 072003 (2006). 
[4] C. Patrignani et al., (Particle Data Group), Chin. Phys. C, 40, 100001 (2016) and 2017 update.

[5] J. Bernabeu, J. Vidal (Valencia U.), G.A. Gonzalez-Sprinberg (Republica U., Montevideo). The Weak magnetic moment of heavy quarks .Feb 1997. 12 pp. Published in Phys.Lett. B397 (1997) 255-262

[6] J. I. Aranda, J. Montaño, F. Ramírez-Zavaleta, J. J. Toscano and E. S Tututi, Phys. Rev. D86, 035008 (2012).

[7] M.A. Arroyo-Ureña, G. Tavares-Velasco, G. Hernández-Tomé. Weak dipole moments of the tau lepton in models with an extended scalar sector. Phys.Rev. D97 (2018) no.1, 013006

[8] J. Bernabéu, G.A. González-Sprinberg, M.Tung and J. Vidal.The tau weak-magnetic dipole moment. Nucl.Phys. B436 (1995) 474-486.

[9] J. Bernabéu, D. Comelli, L. Lavoura, Joao P Silva. Weak magnetic dipole moments in two-Higgs-doublet models. Phys.Rev. D53 (1996) 5222-5232. 